\title{
Treatment cost and life expectancy of diffuse large B-cell lymphoma (DLBCL): a discrete event simulation model on a UK population-based observational cohort
}

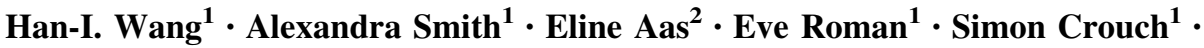 \\ Cathy Burton ${ }^{3} \cdot$ Russell Patmore ${ }^{4}$
}

Received: 3 November 2015/ Accepted: 18 February 2016/Published online: 11 March 2016

(c) The Author(s) 2016. This article is published with open access at Springerlink.com

\begin{abstract}
Background Diffuse large B-cell lymphoma (DLBCL) is the commonest non-Hodgkin lymphoma. Previous studies examining the cost of treating DLBCL have generally focused on a specific first-line therapy alone; meaning that their findings can neither be extrapolated to the general patient population nor to other points along the treatment pathway. Based on empirical data from a representative population-based patient cohort, the objective of this study was to develop a simulation model that could predict costs and life expectancy of treating DLBCL.

Methods All patients newly diagnosed with DLBCL in the UK's population-based Haematological Malignancy Research Network (www.hmrn.org) in 2007 were followed until $2013(n=271)$. Mapped treatment pathways, alongside cost information derived from the National Tariff 2013/14, were incorporated into a patient-level simulation model in order to reflect the heterogeneities of patient characteristics and treatment options. The NHS and social
\end{abstract}

Electronic supplementary material The online version of this article (doi:10.1007/s10198-016-0775-4) contains supplementary material, which is available to authorized users.

Han-I. Wang

han-i.wang@york.ac.uk

1 Epidemiology and Cancer Statistics Group (ECSG), Department of Health Sciences, University of York, Seebohm Rowntree Building, Heslington, York YO10 5DD, UK

2 Department of Health Management and Health Economics, University of Oslo, Oslo, Norway

3 Haematological Malignancy Diagnostic Service, St. James's University Hospital, Leeds, UK

4 Queen's Centre for Oncology and Haematology, Castle Hill Hospital, Hull, UK services perspective was adopted, and all outcomes were discounted at $3.5 \%$ per annum.

Results Overall, the expected total medical costs were $£ 22,122$ for those treated with curative intent, and $£ 2930$ for those managed palliatively. For curative chemotherapy, the predicted medical costs were $£ 14,966, £ 23,449$ and $£ 7376$ for first-, second- and third-line treatments, respectively. The estimated annual cost for treating DLBCL across the UK was around £88-92 million.

Conclusions This is the first cost modelling study using empirical data to provide 'real world' evidence throughout the DLBCL treatment pathway. Future application of the model could include evaluation of new technologies/ treatments to support healthcare decision makers, especially in the era of personalised medicine.

Keywords Diffuse large B-cell lymphoma - DLBCL . Cost - Discrete event simulation · Patient-level simulation

JEL Classification $\quad$ D24 $\cdot$ D61 $\cdot$ E17 $\cdot$ H43 $\cdot$ I1 1

\section{Introduction}

With an annual incidence of around 10.2 per 100,000 in adults, diffuse large B-cell lymphoma (DLBCL) is the commonest lymphoma subtype, accounting for around $40 \%$ of the total $[1,2]$. Although rapidly fatal if left untreated, DLBCL is potentially curable [3]. Introduced in the 1970s, chemotherapy with cyclophosphamide combined with doxorubicin, vincristine and prednisone (CHOP) [4] resulted in a response rate of around $60 \%$ and a long-term cure rate of $30 \%[5,6]$; the addition of the monoclonal antibody rituximab in the 1990s increased the latter to $45 \%$ [7]. 
Hitherto, although several economic studies have been carried out, the majority have focused on comparing the cost-effectiveness of CHOP and R-CHOP (CHOP plus rituximab) [8-17]. In addition, most of the relevant data has come from trials that only include patients treated with curative intent $[9-12,14,16]$; making findings difficult to extrapolate to the general patient population.

The objective of this study was to develop an economic model that could (1) model across the whole treatment pathway, rather than being limited to first-line treatment or a specific agent alone, (2) reflect real world practice rather than the idealized predefined setting of a randomised controlled trial, and (3) predict medical costs and life expectancy. Such a predictive disease model is particularly important for evaluating the cost-effectiveness of new interventions and for allocating health resources efficiently. To the best of our knowledge, no such model has been previously developed for DLBCL.

\section{Method}

\section{Data sources}

The individual-level data used for constructing the simulation model are from a specialist UK population-based registry, the Haematological Malignancy Research Network (www.hmrn.org); the methods of which have been previously described [1, 18]. Briefly, since September 2004, all patients newly diagnosed with a haematological malignancy (leukaemias, lymphomas, and myelomas) in a catchment population of more than 3.6 million have been routinely ascertained and followed-up. HMRN has Sect. 251 support under the NHS Act 2006, which allows all patients regardless of consent, to have full-treatment, response and outcome data collected to clinical trial standards; and to be 'flagged' for death and subsequent cancer registrations at the national Medical Research Information Service (MRIS) and linked to nationwide information on Hospital Episode Statistics (HES).

The current study includes all adult patients ( $\geq 18$ years) newly diagnosed with de novo DLBCL (International Classification of Disease for Oncology, 3rd edition: 9680/3, $9735 / 3,9712 / 3$, and 9679/3) within HMRN in 2007 $(N=271)$. All patients were followed for 5 years from the date of diagnosis, and treatment pathways were individually mapped out according to the chemotherapy regimens received. A more detailed summary of patient characteristics is presented in Supplementary Table 1.

\section{Model structure}

In order to reflect the current treatment strategies, while also being responsive to future changes, a discrete event based micro-simulation model was constructed using Simul8 software (Simul8 2013 Professional version, Simul8 Corporation, Boston, MA, USA). The model first assigned attributes (such as age at diagnosis, sex and prognostic factors) to a group of simulated patients, and then moved each patient forward to the next event, based both on their characteristics and on the timing of the events instead of fixed time cycles.

The model structure was based on patient treatment pathways determined from empirical HMRN data, expert opinion and clinical guidelines. The structure of the model is shown in Fig. 1, and a simplified version of the model can be found via the following link https://www.hmrn.org/ economics/models. Date of diagnosis defines the start of the model; with costs for diagnostic tests such as biopsies, scans, electrocardiography (ECG) and echocardiography (ECHO) being included. After diagnosis, the model splits into two branches according to whether the initial decision was to administer first-line chemotherapy with curative intent or manage supportively using a palliative approach. This is a unique and important feature of the model, ensuring the results reflect 'real world' practice and capture the fact that some patients are managed palliatively from the date of diagnosis until death. For those who entered the first-line curative treatment branch, different chemotherapy regimens, with or without supportive care, were assigned to each patient. The probability of receiving each treatment varied according to the patient's individual attributes [such as age, disease stage and central nervous system (CNS) involvement]. This design allowed the model to capture the differences in 'cost' and 'time in treatment' between alternative regimens. However, it was beyond the scope of this study to compare the economic impact of different first-line chemotherapies.

Once first-line treatment had been received, one of three outcomes was assigned to each individual: died during treatment, responded to treatment, and no response to treatment. The probabilities of these outcomes were dependent on the first-line chemotherapy regimen and age at diagnosis.

For individuals who responded to first-line treatment, one of three events could occur: relapse, remain in remission until cured (defined as staying in remission $\geq 5$ years) and death in remission. For those who were deemed to be cured, it was assumed that mortality returned to that of the 


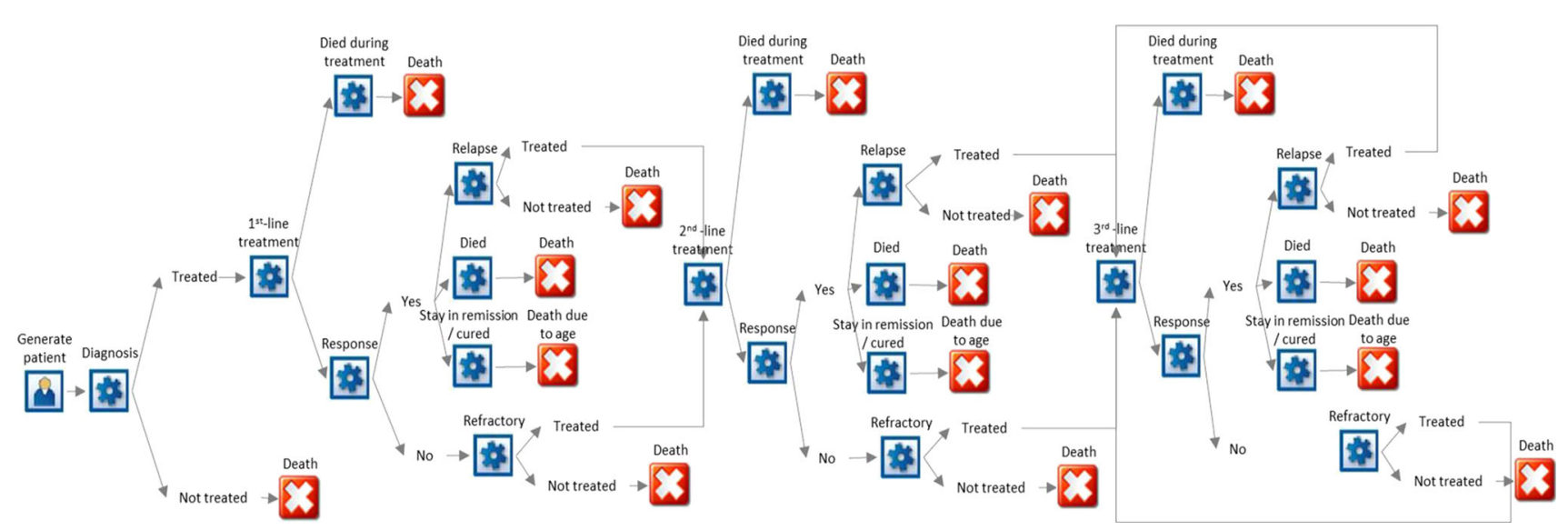

Fig. 1 Model structure

general population and no subsequent DLBCL-related medical costs were incurred.

For individuals who relapsed or had disease that was refractory to treatment, two further options were possible: further potentially curative treatment or the adoption of an end-of-life (palliative) approach. The probability of the decision was dependent on age at diagnosis, previous chemotherapy regimen and response. For those who were not treated curatively, end-of-life care included all care given from last chemotherapy until death. For those who received second-line treatment, different types of chemotherapy regimens with or without autologous stemcell transplant (ASCT) were included. Following this, each patient could remain in remission, receive third-line treatment, or receive end-of-life care; with the decision process being identical to that for first-line treatment. Few patients received treatment post third-line; so for the purposes of the model it was assumed that those who did had similar treatment patterns and response rates to those observed at third line.

The key input parameters used in the model are listed in Tables 1. For more details, please refer to Supplementary Tables 2-4.

\section{Model inputs: medical costs}

The model was built from an NHS perspective; and only medical costs directly related to DLBCL management were considered. This included costs for diagnosis, treatment, supportive care, follow-up and end-of-life care. Details of the cost items and different chemotherapy regimens included in each costing phase can be found in Supplementary Table 5.

All cost parameters were calculated using the National Tariff 2013/14 [19], representing the reimbursement/expenditure of NHS for treating the DLBCL population. For costs that were locally negotiated, such as the costs of chemotherapy regimens, information was derived from the Leeds Teaching Hospital NHS Trust. The inflated NHS reference cost 2012/13 [20] was used only when data were not available. All costs were expressed in 2013 British pound sterling; and the detailed cost information (unit costs) used in the model is summarised in Table 2.

\section{Model inputs: time to event}

Time-to-event (TTE) is a key element for discrete event simulation. Several time-to-event analyses were carried out using empirical data derived from HMRN to estimate the distributions associated with time between two events. This included the time from diagnosis to treatment, time in treatment, time from response to relapse, time from response to death and time in end-of-life care. All time-toevent analyses (survival analyses) were based on the best fit distributions as a function of patient's age, treatment intent and treatment details. Five parametric survival models (exponential, Weibull, log-normal, log-logistic and Gompertz distributions) were tested and the best fit model was determined using the Akaike information criterion (AIC) score. It was assumed that cured patients' mortality would return to normal, and the distribution of time to death was generated using the United Kingdom National Life Table, 2011-2013 [21]. The key parameters used in the model are illustrated in Fig. 2a-d. For more details on the time-toevent analyses, please refer to Supplementary Table 6.

\section{Model outputs}

Health outcome was measured by life-years gained (LYG), while economic outcomes were captured by medical costs. Both economic and health outcomes were discounted using a $3.5 \%$ annual discount rate, based on UK guidance recommended by the National Institute for Health and Clinical Excellence (NICE) [22]. 
Table 1 Key model parameters

\begin{tabular}{l}
\hline Parameters \\
\hline Patient generation state \\
Age (years) \\
Sex \\
Age $\leq 40$ \\
Age $40-50$ \\
Age $50-60$ \\
Age $60-70$ \\
Age 70-80 \\
Age $\geq 80$ \\
Diagnosis \\
Diagnostic test results \\
Age $\leq 40$
\end{tabular}

Stage IA: 0.06

CNS involvement: 0.0

Standard: 0.94

Died before diagnosis: 0.0

Age 40-50

Age 50-60

Age 60-70

Age 70-80

Age $\geq 80$

Male: 0.56

Male: 0.68

Male: 0.71

Male: 0.50

Male: 0.55

Male: 0.51

Stage IA: 0.09

CNS involvement: 0.09

Standard: 0.77

Died before diagnosis: 0.05

Stage IA: 0.15

CNS involvement: 0.08

Standard: 0.75

Died before diagnosis: 0.02

Stage IA: 0.06

CNS involvement: 0.02

Standard: 0.90

Died before diagnosis: 0.02

Stage IA: 0.12

CNS involvement: 0.01

Standard: 0.75

Died before diagnosis: 0.12

Stage IA: 0.05
CNS involvement: 0.00

Standard: 0.89

Died before diagnosis: 0.06

Initial treatment decision

$\begin{array}{ll}\text { Age } \leq 40 & \text { Treated: } 0.94 \\ \text { Age } 40-50 & \text { Treated: } 0.95 \\ \text { Age } 50-60 & \text { Treated: } 0.96 \\ \text { Age } 60-70 & \text { Treated: } 0.88 \\ \text { Age } 70-80 & \text { Treated: } 0.75 \\ \text { Age } \geq 80 & \text { Treated: } 0.54\end{array}$

Treated: 0.54

Treatment decision for

$\begin{array}{ll}\text { Age } \leq 60 & \text { Treated: } 0.86 \\ \text { Age } 60-80 & \text { Treated: } 0.33 \\ \text { Age } \geq 80 & \text { Treated: } 0.00\end{array}$

Beta

Mean (sd): 67.8 (14.7)

Beta $(3.73,2.32)$

Beta

$\alpha=9, \beta=7$

Beta

$\alpha=15, \beta=7$

Beta

$\alpha=29, \beta=12$

Beta

$\alpha=31, \beta=31$

Beta

$\alpha=42, \beta=35$

Beta

$\alpha=27, \beta=26$

Dirichlet

$\alpha_{1}=2$

$\alpha_{2}=1$

$\alpha_{3}=16$

$\alpha_{4}=2$

Dirichlet

$\alpha_{1}=3$

$\alpha_{2}=3$

$\alpha_{3}=18$

$\alpha_{4}=2$

Dirichlet $\quad \alpha_{1}=7$

$\alpha_{2}=4$

$\alpha_{3}=32$

$\alpha_{4}=2$

Dirichlet $\quad \alpha_{1}=5$

$\alpha_{2}=2$

$\alpha_{3}=57$

$\alpha_{4}=2$

Dirichlet

$\alpha_{1}=10$

$\alpha_{2}=2$

$\alpha_{3}=59$

$\alpha_{4}=10$

$\alpha_{1}=4$

$\alpha_{2}=1$

$\alpha_{3}=48$

$\alpha_{4}=4$

Beta

$\alpha=15, \beta=1$

$\alpha=21, \beta=1$

$\alpha=39, \beta=2$

$\alpha=55, \beta=7$

$\alpha=58, \beta=19$

$\alpha=29, \beta=24$

$\alpha=6, \beta=1$

$\alpha=4, \beta=8$

$\alpha=0, \beta=6$ 
Table 1 continued

\begin{tabular}{|c|c|c|c|}
\hline Parameters & Estimates & \multicolumn{2}{|c|}{ Distribution } \\
\hline \multicolumn{4}{|c|}{ Treatment decision for patients relapsed following first-line chemotherapy } \\
\hline Age $\leq 60$ & Treated: 0.93 & Beta & $\alpha=14, \beta=1$ \\
\hline Age $60-80$ & Treated: 0.53 & Beta & $\alpha=8, \beta=7$ \\
\hline Age $\geq 80$ & Treated: 1.00 & Beta & $\alpha=2, \beta=0$ \\
\hline \multicolumn{4}{|c|}{ Treatment decision for patients refractory to second-line chemotherapy } \\
\hline Age $\leq 60$ & Treated: 0.50 & Beta & $\alpha=4, \beta=4$ \\
\hline Age $60-80$ & Treated: 0.50 & Beta & $\alpha=3, \beta=3$ \\
\hline Age $\geq 80$ & Treated: 0.00 & Beta & $\alpha=0, \beta=1$ \\
\hline \multicolumn{4}{|c|}{ Treatment decision for patients relapsed following second-line chemotherapy } \\
\hline Age $\leq 60$ & Treated: 0.50 & Beta & $\alpha=1, \beta=1$ \\
\hline Age $60-80$ & Treated: 0.00 & Beta & $\alpha=0, \beta=1$ \\
\hline Age $\geq 80$ & Treated: 0.00 & Beta & $\alpha=0, \beta=1$ \\
\hline \multicolumn{4}{|c|}{ Treatment decision for patients refractory to third-line chemotherapy } \\
\hline Age $\leq 60$ & Treated: 0.50 & Beta & $\alpha=0, \beta=3$ \\
\hline Age $60-80$ & Treated: 0.00 & Beta & $\alpha=0, \beta=1$ \\
\hline Age $\geq 80$ & Treated: $0.00^{\mathrm{a}}$ & Beta & $\alpha=0, \beta=1$ \\
\hline \multicolumn{4}{|c|}{ Treatment decision for patients relapsed following third-line chemotherapy } \\
\hline Age $\leq 60$ & Treated: $0.50^{\mathrm{a}}$ & Beta & $\alpha=1, \beta=1$ \\
\hline Age $60-80$ & Treated: $0.00^{\mathrm{a}}$ & Beta & $\alpha=0, \beta=1$ \\
\hline Age $\geq 80$ & Treated: $0.00^{\mathrm{a}}$ & Beta & $\alpha=0, \beta=1$ \\
\hline
\end{tabular}

CNS central nervous system

a The probability was assumed to be the same as the probability in the second line due to lack of data

\section{Assessing uncertainty}

Probabilistic sensitivity analysis was performed on all parameters in order to explore the cumulative uncertainty of the model. Each parameter was assigned a distribution to reflect sample variability, whilst coefficients of survival models were assigned multivariate normal distributions. Then, Monte Carlo simulations were carried out by sampling parameters from the corresponding distributions simultaneously over a large number of iterations until stable results were reached (500 times). All outputs from the iterations were summarised with $95 \%$ confidence intervals [23].

\section{Analysis}

To investigate the impact on the UK as a whole, the annual number of expected cases in the UK $(N=4880)$ derived from HMRN rates was used to run the simulation model www.hmrn.org/statistics/incidence. Incidence-based results were presented in aggregate, as well as for the time horizons of 5-year, 15-year, and lifetime (simulated until 100 years of age or death). Survival beyond 5 years was extrapolated based on the best fit time-to-event distributions derived from the empirical data and the UK National Life Table, 2011-2013 [21]. See "Model inputs: time to event" in Methods for details.

To further investigate the effect of age, a sub-simulation was conducted to capture differences in cost and life-years gained for two age groups: under 70 and over 70 years of age. In addition, using the expected number of new cases of DLBCL diagnosed each year in the UK $(N=4880)$, the model further simulated national prevalence-based costs and life-years gained. Results were collected after a burnin period of 10 years.

\section{Validation}

The model was validated by means of standard methods, including face, internal and external validations [24]. Face validation was conducted while the model was under construction by consulting clinical experts on model structure, data sources and results. Internal validation was assessed by comparing predicted costs and life-years gained with empirical estimates, and external validation was carried out by comparing simulated results with relevant literature. 
Table 2 Summary of key unit costs

\begin{tabular}{|c|c|c|}
\hline & Unit cost & Source \\
\hline \multicolumn{3}{|l|}{ Inpatient stay } \\
\hline Spell cost & $£ 797$ & National Tariff 2013/14 \\
\hline Cost per excess bed day & $£ 243$ & National Tariff 2013/14 \\
\hline \multicolumn{3}{|l|}{ Outpatient visit } \\
\hline First attendance (single profession) & $£ 247$ & National Tariff 2013/14 \\
\hline First attendance (multi-profession) & $£ 248$ & National Tariff 2013/14 \\
\hline Follow-up visit (single profession) & $£ 113$ & National Tariff 2013/14 \\
\hline Follow-up visit (multi-profession) & $£ 174$ & National Tariff 2013/14 \\
\hline \multicolumn{3}{|l|}{ Diagnostic procedures } \\
\hline Diagnostic biopsy & $£ 260$ & National Tariff $2013 / 14^{\mathrm{a}}$ \\
\hline Staging biopsy ${ }^{\mathrm{b}}$ & $£ 503$ & National Tariff 2013/14 \\
\hline \multicolumn{3}{|l|}{ Imaging } \\
\hline Computed tomography (CT) & $£ 105$ & National Tariff 2013/14 \\
\hline Magnetic resonance imaging (MRI) & $£ 206$ & National Tariff 2013/14 \\
\hline Positron emission tomography (PET) & $£ 748$ & National Tariff 2013/14 \\
\hline Ultrasound & $£ 51$ & National Tariff 2013/14 \\
\hline Electrocardiography (ECG) & $£ 172$ & National Tariff 2013/14 \\
\hline Echocardiography (Echo) & $£ 322$ & National Tariff 2013/14 \\
\hline \multicolumn{3}{|l|}{ Radiotherapy } \\
\hline Planning & $£ 769$ & National Tariff 2013/14 \\
\hline Per fraction & $£ 123$ & National Tariff 2013/14 \\
\hline \multicolumn{3}{|l|}{ Chemotherapy (per cycle) } \\
\hline $\mathrm{CHOP}$ & $£ 289$ & Leeds Teaching Hospitals Trust \\
\hline $\mathrm{R}-\mathrm{CHOP}$ & $£ 1730$ & Leeds Teaching Hospitals Trust \\
\hline $\mathrm{R}-\mathrm{CVP}$ & $£ 1486$ & Leeds Teaching Hospitals Trust \\
\hline CODOX-M/IVAC-R & $£ 6241$ & Leeds Teaching Hospitals Trust \\
\hline IDARAM & $£ 2006$ & Leeds Teaching Hospitals Trust \\
\hline R-DHAP & $£ 1952$ & Leeds Teaching Hospitals Trust \\
\hline R-ESHAP & $£ 3344$ & Leeds Teaching Hospitals Trust \\
\hline MiniBEAM & $£ 446$ & Leeds Teaching Hospitals Trust \\
\hline Methotrexate (low dose intrathecal) & $£ 5$ & Leeds Teaching Hospitals Trust \\
\hline Methotrexate (high dose) & $£ 861$ & Leeds Teaching Hospitals Trust \\
\hline Autologous stem-cell transplant (ASCT) & $£ 42,000$ & Leeds Teaching Hospitals Trust \\
\hline
\end{tabular}

CHOP cyclophosphamide, doxorubicin, vincristine and prednisone, $R$-CHOP cyclophosphamide, doxorubicin, vincristine, prednisone and rituximab, $R-C V P$ cyclophosphamide, vincristine, prednisone and rituximab, CODOX-M/IVAC-R cyclophosphamide, doxorubicin, vincristine, methotrexate/ifosfamide, etoposide, high dose cytarabine and rituximab, IDARAM rituximab, idarubicin, dexamethasone, cytarabine and methotrexate, R-DHAP dexamethasone, cytarabine, cisplatin and rituximab, R-ESHAP etoposide, methylprednisolone, cytarabine, cisplatin and rituximab, MiniBEAM carmustine, etoposide, cytarabine and melphalan

a Average of National Tariffs 2013/14

b Including: BMAT bone marrow aspirate and trephine, $B M A$ bone marrow aspirate and $T B$ bone marrow trephine

\section{Results}

\section{Incidence-based results}

Predicted costs, as well as life-days gained over 5-year, 15-year, and lifetime horizons are presented in Table 3 according to the simulation results of the model. Results for the 5-year horizon provide meaningful clinical estimates and allow internal validation, and results for the lifetime horizon provide insight into the overall economic and health impacts throughout the treatment pathway. Results over a 15-year time horizon are presented for the 

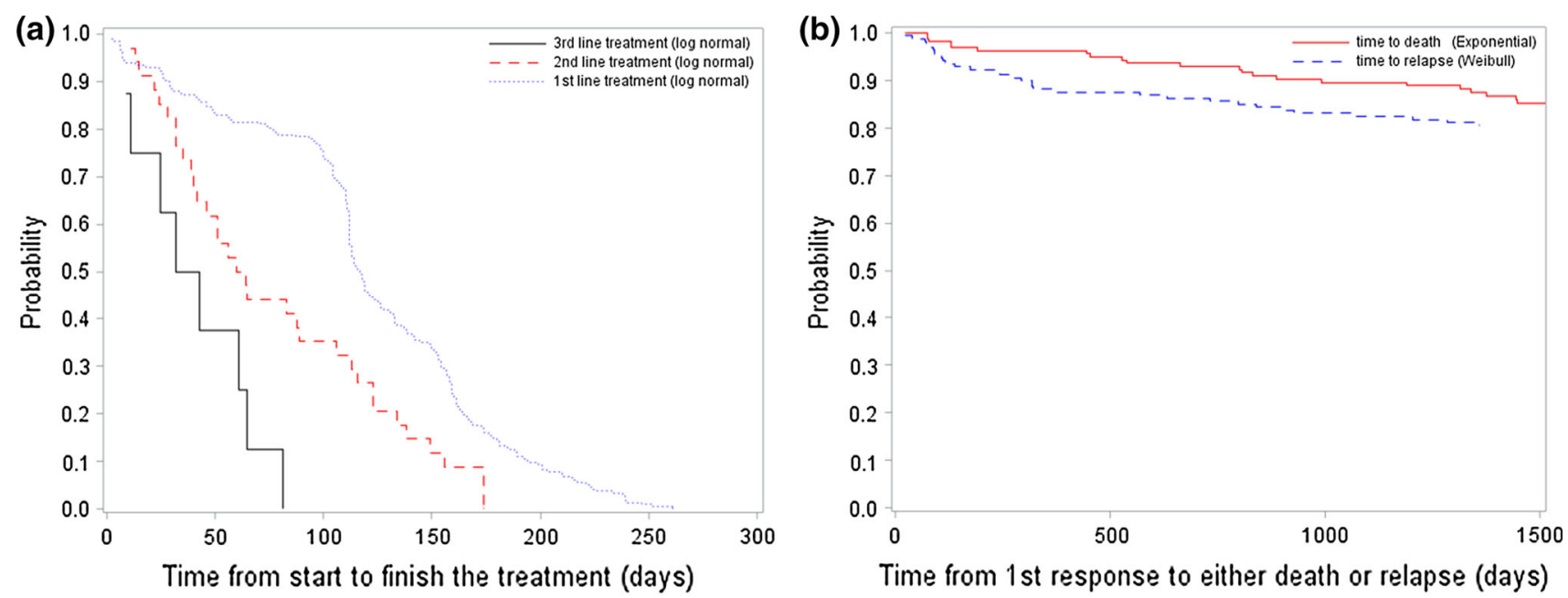

Time from 1 st response to either death or relapse (days)
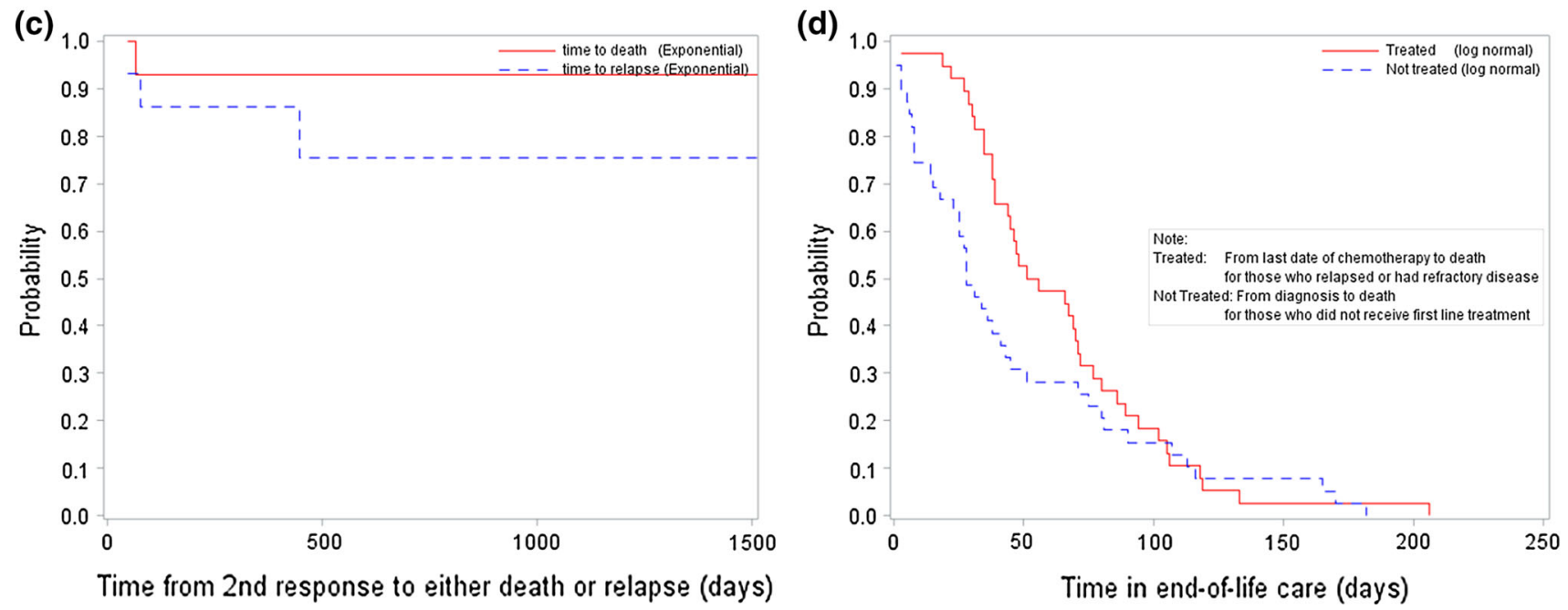

Fig. 2 Time to event analyses. a Time in treatment. b Time in first response to either death or relapse. $\mathbf{c}$ Time in second response to either death or relapse. d Time in end-of-life care

purpose of external comparison, as most published estimates are for this time period.

Overall, the average cost per patient was around $£ 18,000$. This figure is consistent regardless of the time horizon chosen $(£ 18,096, £ 18,396$ and $£ 18,396$ for 5 -year, 15 -year and lifetime, respectively). This reflects the fact that for most DLBCL patients, treatment is completed within the 5-year time frame. However, the predicted lifeyears gained varied with time horizon: being 2.8, 6.3 and 10.0 for 5-year, 15-year and lifetime, respectively.

As expected, the costs for patients who received treatment with curative intent were significantly higher (£22,122), with more life-years gained (12.6 LYG) than for patients who were not treated (£2930, 0.1 LYG). This also applied to patients who received treatment post secondline. For patients who received ASCT as second-line treatment, costs were higher, but longer survival was observed (19.9 LYG and 7.6 LYG for ASCT and nonASCT at second-line, respectively).

Table 4 shows the cost and time-to-next-event components of the overall treatment pathway over the lifetime horizon. As shown, treatment cost is the main component of the total costs. This is particularly prominent for secondline treatment involving ASCT $(£ 56,442)$. For end-of-life care, patients who were not treated incurred less costs (£2930) than those who were treated prior to receiving endof-life care (£4767).

\section{Sub-group analysis}

Figure 3 shows the effect of age for subgroups with different initial treatment intents. As expected, patients younger than 70 years had better survival but incurred more medical costs than those aged 70 years or more. 


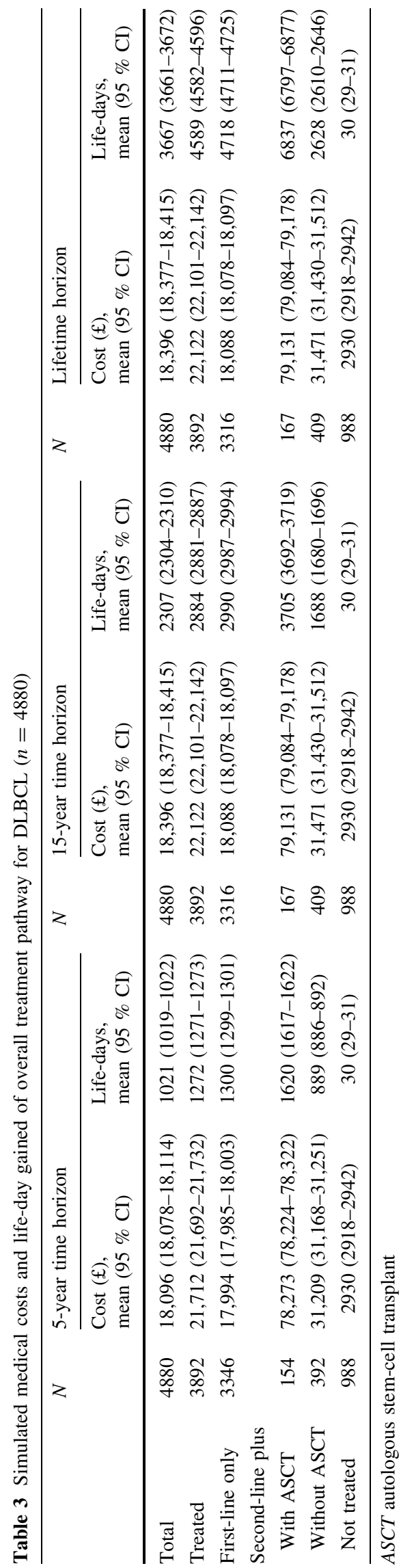

However, for those who did not receive treatment with curative intent, medical costs and survival were similar between the two age groups.

Figure 3 also demonstrates the effect of time-horizon choice. As shown in Fig. $3 \mathrm{~b}$ and e, among treated patients, survival differed between the two age groups (6255 and 2080 days over the lifetime). However, over a 5-year time horizon, the differences in life-years gained and costs were much smaller (1408 vs 1065 days); confirming that patients who were over the age of 70 responded as well as those who were younger.

\section{Prevalence-based results}

The prevalence-based cost demonstrates the total cost associated with treating existing and new DLBCL patients during a 1-year period. The results are summarised in Fig. 4. As shown, the total annual costs for treating the DLBCL patient population across the UK as a whole was in the region of $£ 88$ million for the lower bound and $£ 92$ million for the upper bound.

\section{Model validation}

With respect to face validity, the model structure, data source, and results were corroborated by consultant haematologists (CB and RP). For internal validity, the simulated/predicted outcomes were compared to empirical estimates derived from HMRN. The survival curves over a 5-year period are compared in Fig. 5. As shown, the simulated results closely match the 5-year follow-up empirical data, demonstrating good fit with empirical evidence. With regard to medical costs, the average 5-year simulated cost was $£ 18,096$ per patient (ranging from $£ 18,078$ to $£ 18,114$ among 500 iterations), capturing $98 \%$ of the empirical results derived from the study population $(£ 18,515)$.

Regarding external validity, the simulated cost and survival results were broadly similar to the findings in earlier literature $[8,10,16,17]$. Taking the average costs of first-line treatment with R-CHOP as an example: for those over 60 years of age, our 5-year result of $£ 20,831$ is close to the US estimate of $£ 19,485$ [16] and the UK estimate of $£ 19,805$ [10], but less than the Canadian estimate of $£ 26,498$ [8]. For those under 60 years of age, our 15 -year result of $£ 26,761$ is close to the Canadian estimate of $£ 28,626$ [8], but more than the UK estimate of $£ 20,798$ [10] (all currencies were inflated and converted to 2013 British pound sterling). Regarding the average survival time following R-CHOP, for those over 60 years of age, our 15 -year result of 7.87 years is close to the estimate of 6.2 years from Johnston's study [8] and the estimate of 6.23 years from Knight's study [10]. For those under 60 years of age, our 15-year result of 11.8 years is slightly 
Table 4 Costs and time-toevent breakdowns for treated DLBCL patients (based on lifetime horizon)

\begin{tabular}{|c|c|c|c|}
\hline & $N$ & $\begin{array}{l}\text { Cost }(\mathfrak{f}) \\
\text { Mean }(95 \% \text { CI })\end{array}$ & $\begin{array}{l}\text { Duration of event (days) } \\
\text { Mean }(95 \% \mathrm{CI})\end{array}$ \\
\hline Diagnosis & 4880 & $1326(1325-1327)$ & - \\
\hline \multicolumn{4}{|l|}{ Treatment } \\
\hline First-line treatment & 3892 & $14,966(14,958-15,974)$ & $122(121-123)$ \\
\hline Second-line treatment & 577 & $23,449(23,365-23,534)$ & $81(80-82)$ \\
\hline With ASCT & 167 & $56,442(56,409-56,474)$ & $104(103-105)$ \\
\hline Without ASCT & 409 & 9956 (9932-9981) & $72(71-73)$ \\
\hline Third-line treatment & 106 & $7376(7374-7406)$ & $50(49-51)$ \\
\hline \multicolumn{4}{|l|}{ Follow-up } \\
\hline During first response & 3001 & $1401(1400-1402)$ & $5125(5117-5132)$ \\
\hline $\begin{array}{l}\text { During second } \\
\text { response }\end{array}$ & 296 & 1371 (1369-1374) & $6135(6106-6163)$ \\
\hline \multicolumn{4}{|l|}{ End-of-life care } \\
\hline For not treated patients & 988 & 2930 (2918-2942) & $30(29-31)$ \\
\hline For treated patients & 704 & $4767(4755-4780)$ & $60(60-61)$ \\
\hline
\end{tabular}

ASCT autologous stem-cell transplant more than Knight's estimate of 9.9 years [10] and Johnston's estimate of 8.3 years [8]. Thus, overall, our model demonstrated good capability for predicting both medical costs and life expectancy. For more details, please refer to online Supplementary Table 7.

\section{Discussion}

This is the first DLBCL model to simulate and predict treatment costs and life-years gained throughout the treatment pathway. Whilst several economic models have previously been constructed and published for DLBCL, all were built for the purpose of assessing the cost-effectiveness of adding rituximab to CHOP, not for overall disease treatments [8-10, 14-16]. Hence, although these reports confirmed the fact that adding rituximab to CHOP was cost-effective, none could examine the overall economic impact to health insurers or policy makers. In addition, this is the first DLBCL model to incorporate data on patients who were not treated with curative intent; enabling the production of more accurate estimates of the economic impact of DLBCL among different patient groups, as well as across the patient population as a whole. This flexibility allowed quantities such as the prevalence-based economic impact at a national level to be explored for the first time.

In the current study, a discrete event simulation (DES) model was built for analysis. The DES model generated individual treatment histories within the set time horizon for hypothetical DLBCL patients based on 'real-world' observational data. Estimates for the 5-year expected medical cost and life-years gained were $£ 18,096$ and 2.8 years, respectively, while the lifetime expected medical cost and survival were $£ 18,396$ and 10.0 years, respectively. Curative treatment results in a high number of lifeyears gained at the relatively moderate cost of $£ 1535$ per life-year gained $(95 \%$ CI $£ 1534-£ 1537$ per life-year gained) compared to non-curative care only. The variability was driven largely by initial treatment, age at diagnosis, and whether the patient had an ASCT. The expected lifetime medical cost ranged from $£ 79,131$ to $£ 2930$, while the life expectancy ranged from 30 days to 19 years (Table 3 ).

With respect to appropriate time horizons, this study demonstrated that the differences in estimated costs derived from 5-year, 15-year, and lifetime horizons were relatively minor, reflecting the high response rate amongst treated patients. However, the differences in estimated lifeyears gained were considerable (Table 3); confirming, as has been suggested by others [14], that the lifetime horizon is the optimal approach as it allows the overall effects of treatment to be fully captured. Furthermore, as expected, patients younger than 70 years had better survival and therefore incurred higher medical costs (Fig. 3). Importantly, however, in the 5-year time frame the differences in costs and life-years gained between these two age groups were small, demonstrating that patients over 70 years who receive chemotherapy responded as well as their younger counterparts. In this context, it is important to note that a patient's performance status has been shown to be more discriminatory of survival than chronological age [25]. Finally, although the average cost of treating DLBCL is considered moderate in comparison to some other cancers ( $£ 18,396$, see Table 1 ), the annual economic impact of treating existing and new DLBCL patients in the UK is considerable (in the region of $£ 88-£ 92$ million, see Fig. 4); accounting for approximately $1 / 6$ th of the annual UK NHS 
Life time horizon

(based on 500 model runs)

(a)

Overall

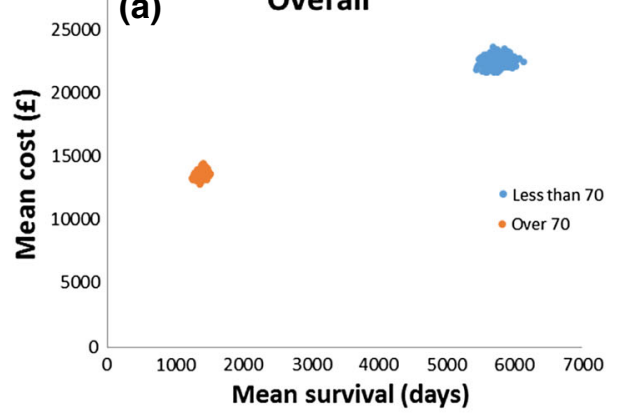

(b) Treated patients

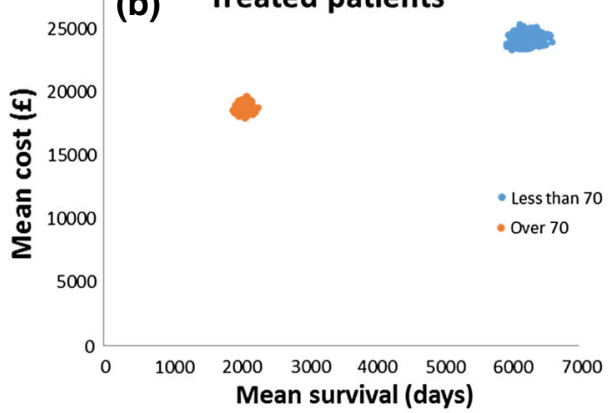

(c) Not treated patients

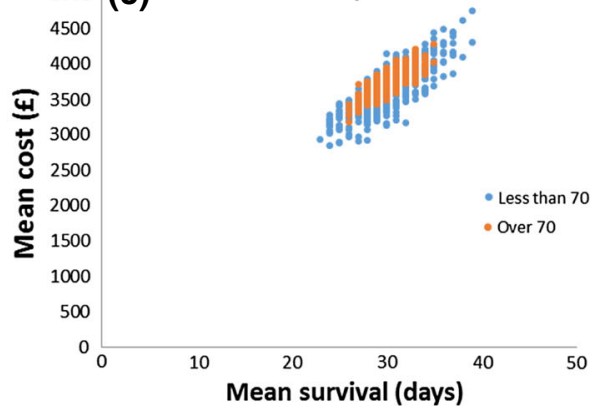

Five-year time horizon

(based on 500 model runs)

(d)

Overall

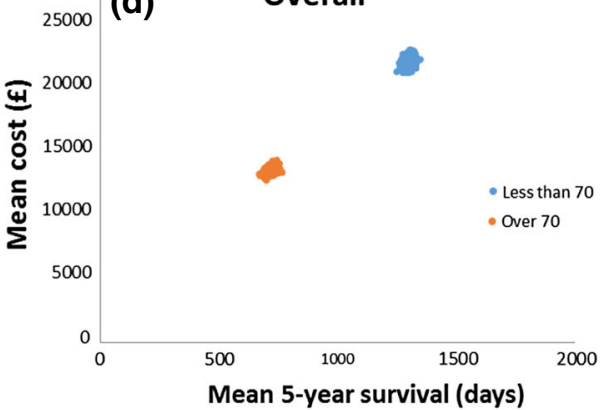

(e) Treated patients
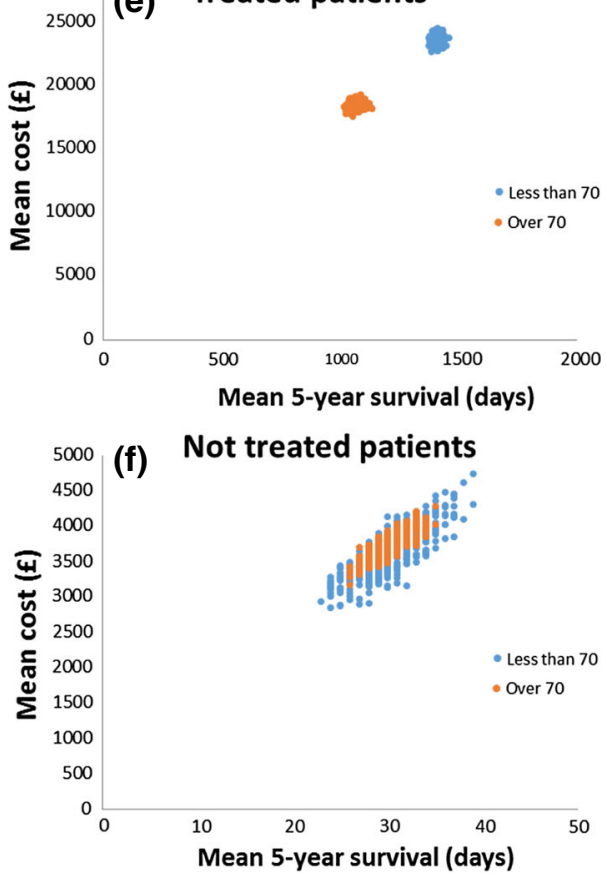

\begin{tabular}{|c|c|c|c|c|c|c|}
\hline & $\mathbf{N}$ & Mean cost $(£)$ & $\begin{array}{l}\text { Mean survival } \\
\text { (days) }\end{array}$ & $\mathbf{N}$ & Mean cost & $\begin{array}{c}\text { Mean 5-year } \\
\text { survival (days) }\end{array}$ \\
\hline \multicolumn{7}{|l|}{$\leq 70$ years } \\
\hline Overall & 2541 & $22,665(22,637-22,693)$ & $5,761(5,751-5,770)$ & 2541 & $22,162(22,135-22,190)$ & $1,299(1,298-1,300)$ \\
\hline Treated & 2340 & $24,306(24,276-24,335)$ & $6,255(6,245-6,265)$ & 2340 & $23,759(23,730-23,788)$ & $1,408(1,407-1,410)$ \\
\hline Not Treated & 202 & $3,632(3,604-3,659)$ & $30(29-31)$ & 202 & $3,632(3,604-3,659)$ & $30(29-31)$ \\
\hline \multicolumn{7}{|l|}{$>70$ years } \\
\hline Overall & 2339 & $13,757(13,736-13,778)$ & 1,391 (1,387-1,395) & 2339 & $13,622(13,601-13,643)$ & 717 (716-719) \\
\hline Treated & 1553 & $18,830(18,806-18,854)$ & $2,080(2,075-2,085)$ & 1553 & $18,627(18,603-18,650)$ & $1,065(1,064-1,067)$ \\
\hline Not Treated & 786 & $3,734(3,720-3,748)$ & $30(30-31)$ & 786 & $3,734(3,720-3,748)$ & $30(30-31)$ \\
\hline
\end{tabular}

Fig. 3 Simulated cost and survival between two age groups for three groups: a Overall. b Patients who received initial treatments and c patients who did not receive initial treatment over lifetime horizon,

budget for haematological diseases as a whole [26] and providing around 35,000 life-years gained per year (data not shown).

Predicated on 'real-world' data, this model produced findings that can be extrapolated to the general patient and $\mathbf{d}$ overall. e Patients who received initial treatments and $\mathbf{f}$ patients who did not receive initial treatment over 5 -year time horizon

population; which is not the case for models built using data from clinical trials $[9-12,14,16]$. The reliability and robustness of the model are also confirmed by the internal and external validations. For internal validation, the simulated cost and life-year results were close to the empirical 
Fig. 4 Annual cost (prevalence-based cost) for treating DLBCL population across the UK as a whole

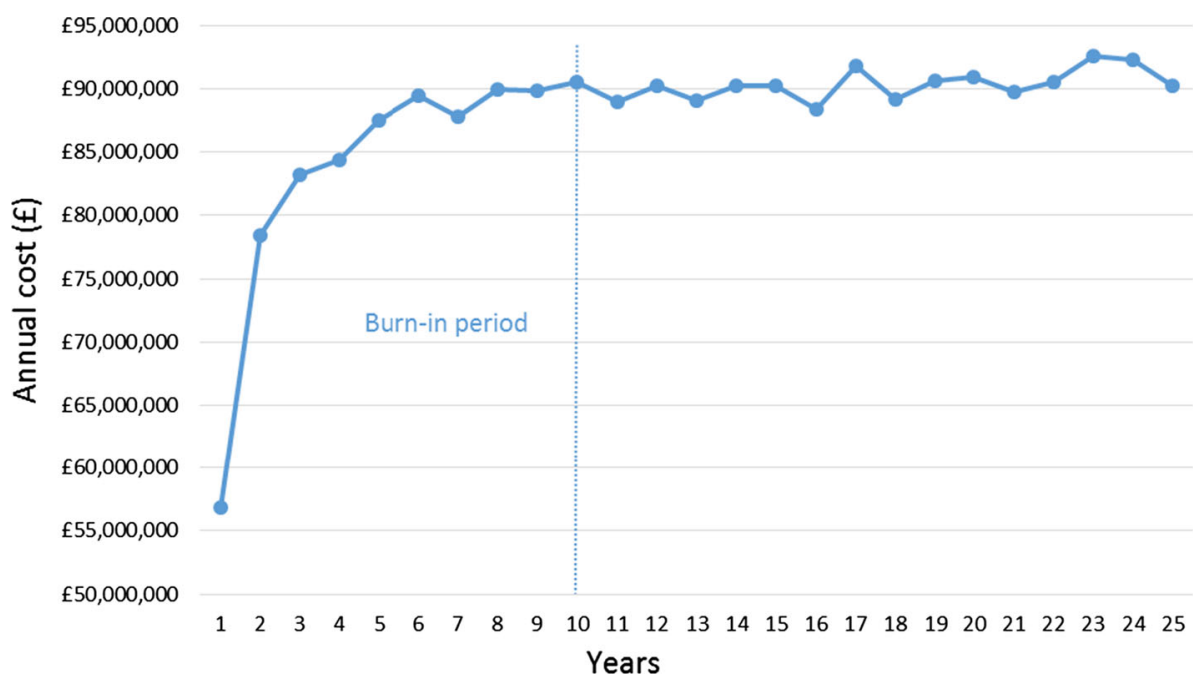

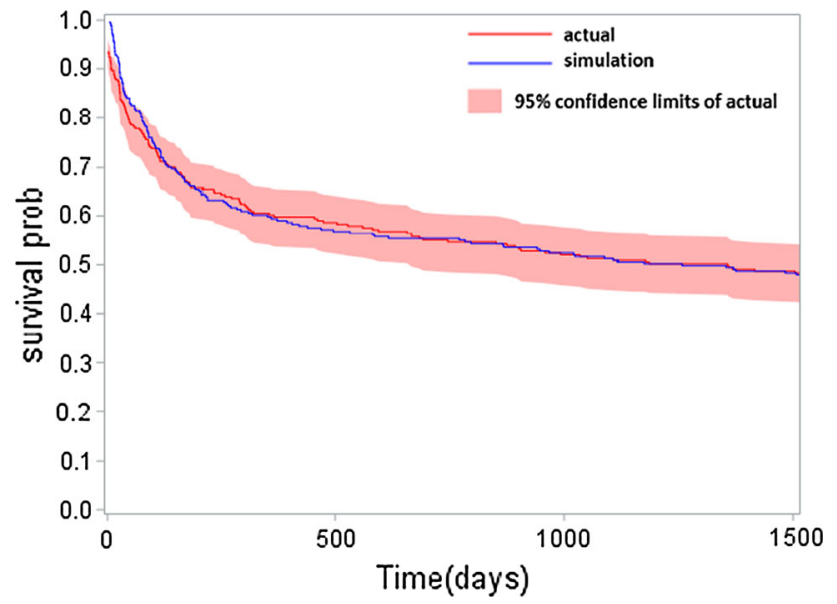

Fig. 5 Survival curves of empirical and probabilistic models

HMRN data (Fig. 5). For external validation, the model results were in line with the findings from other observational studies: the micro-costing study conducted in Canada [17] and the Medicare claims study conducted in the US [16]. Moreover, the structure of our DES model provided an opportunity to make detailed comparisons with findings from more restricted datasets: and when the parameters (e.g. specific age groups, chemotherapy regimens and time horizons) from those studies were applied to the model, the results were found to be in line with most of the relevant studies $[8,10,16,17]$ (please refer to "Model validation").

The molecular heterogeneity of DLBCL coupled with recent advances in diagnostic technologies [27, 28] is resulting in the development of more targeted approaches to the treatment of this complex cancer. The recent UK Phase 3 trial (REMoDL-B), for example, used gene expression profiling to assign DLBCL patients to different treatment arms [29]; and, as science continues to advance and new treatments emerge, reliable models such as the one developed here will become increasingly important in this rapidly changing field. Furthermore, the reduced sideeffects and toxicities of many of these novel therapeutic agents mean that the proportion of the patient population who receive treatment will continue to increase. Accordingly, our model's ability to accurately predict the impact of these changes across the entire population of patients with DLBCL will not only support commissioners to allocate resources, but will also aid clinical decision making.

With respect to model limitations, the current study did not deal with an administrative censoring effect. However, this is unlikely to impact on results, as the degree of administrative censoring is non-informative (independent of treatment). Also, the model is validated by the results which are broadly similar to the findings in earlier literature. In addition, whilst cost inputs were mainly confined to inpatient and day-case settings, the majority of costs will have been captured since around $85 \%$ of cancer spending is incurred in acute/secondary care settings [26]. Moreover, as the model is based on an empirical follow-up time of only 5 years, the results for treated patients need to be interpreted with caution. Nonetheless, as the majority of treatments are carried out within the first 5 years, the costs estimates will be largely unaffected (see Table 3 ). It is, however, worth noting that our long-term estimates are conservative since they do not include health care costs for comorbidities that could potentially have been caused by DLBCL therapy. Quality adjusted life years (QALY) were not used in the model, as this information was not available for this cohort of patients and currently no other high quality QALY information is published in the existing literature, an issue recognised by other researchers $[10,15]$. 
However, the model has been built to allow QALY data to be incorporated in the future and we are now collecting European Quality of Life-5 Dimensions (EQ-5D) information from patient cohort members.

\section{Conclusion}

Life expectancy and costs of treating DLBCL patients vary according to patient characteristics and treatment pathways. However, the population-based model developed in the current study demonstrated a good capability of capturing the medical costs to healthcare commissioners, as well as the life-years gained in a real world setting. Importantly, the model produces different outputs for different purposes; estimating total costs along with health benefits at varying time points for specific patient cohorts, as well as generating prevalence-based costs for all patients over specific time periods. Future application of the model could include evaluation of new technologies/treatments to support healthcare decision makers, especially in the era of personalised medicine.

Acknowledgments The Haematological Malignancy Research Network is funded by Bloodwise. It has ethics approval (REC 04/01/ 1205/69) from Leeds West Research Ethics Committee. R\&D approval from each NHS Trust in the study area and exemption from Sect. 251 of the Health and Social Care Act (PIAG 1-05(h)/2007). This work was carried out as part of a joint working project with Roche.

\section{Compliance with ethical standards}

Conflict of interest This study was funded by Bloodwise. The authors: Han-I Wang, Alexandra Smith, Eline Aas, Eve Roman, Simon Crouch, Cathy Burton, and Russell Patmore have no conflicts of interest.

Open Access This article is distributed under the terms of the Creative Commons Attribution 4.0 International License (http://crea tivecommons.org/licenses/by/4.0/), which permits unrestricted use, distribution, and reproduction in any medium, provided you give appropriate credit to the original author(s) and the source, provide a link to the Creative Commons license, and indicate if changes were made.

\section{References}

1. Smith, A., Roman, E., Howell, D., Jones, R., Patmore, R., Jack, A.: The Haematological Malignancy Research Network (HMRN): a new information strategy for population based epidemiology and health service research. Br. J. Haematol. 148, 739-753 (2010)

2. Haematological Malignancy Research Network. https://www. hmrn.org/statistics/incidence

3. Devita, V., Canellos, G., Chabner, B., Schein, P., Hubbard, S., Young, R.: Advance diffuse histiocytic lymphoma, a potentially curable disease. Lancet. 305, 248-250 (1975)
4. Yi, P.I., Coleman, M., Saltz, L., Norton, L., Topilow, A.A., Adler, K., Bernhardt, B.: Chemotherapy for large cell lymphoma: a status update. Semin. Oncol. 17, 60-73 (1990)

5. Coltman, C., Dahlberg, S., Jones, S.: CHOP is curative in thirty percent of patients with large cell lymphoma: a twelve-year Southwest Oncology Group follow-up. In: Advances in cancer chemotherapy. pp. 71-78. Park Row, New York (1986)

6. Kimby, E., Brandt, L., Nygren, P., Glimelius, B.: SBU-group. Swedish Council of Technology Assessment in Health Care: a systematic overview of chemotherapy effects in aggressive nonHodgkin's lymphoma. Acta Oncol. 40, 198-212 (2001)

7. Keegan, T.H.M., Moy, L.M., Foran, J.M., Alizadeh, A.A., Chang, E.T., Shema, S.J., Schupp, C.W., Clarke, C.A., Glaser, S.L.: Rituximab use and survival after diffuse large B-cell or follicular lymphoma: a population-based study. Leuk. Lymphoma 54, 743-751 (2013)

8. Johnston, K.M., Marra, C.A., Connors, J.M., Najafzadeh, M., Sehn, L., Peacock, S.J.: Cost-effectiveness of the addition of rituximab to $\mathrm{CHOP}$ chemotherapy in first-line treatment for diffuse large B-cell lymphoma in a population-based observational cohort in British Columbia, Canada. Value Health. 13, 703-711 (2010)

9. Ferrara, F., Ravasio, R.: Cost-effectiveness analysis of the addition of rituximab to CHOP in young patients with good-prognosis diffuse large-B-cell lymphoma. Clin. Drug Investig. 28, 55-65 (2008)

10. Knight, C., Hind, D., Brewer, N., Abbott, V.: Rituximab (MabThera) for aggressive non-Hodgkin's lymphoma: systematic review and economic evaluation. Health Technol Assess. 8, iii, ix-xi, 1-82 (2004)

11. Knight, C., Maciver, F.: The cost-effectiveness of rituximab in non-Hodgkin's lymphoma. Expert Rev Pharmacoecon Outcomes Res. 7, 319-326 (2007)

12. Lupu, A., Radu, P., Pană, B., Kalfas, C.: R-CHOP vs. CHOP: a cost-effectiveness analysis on aggressive non-Hodgkin's lymphoma (NHL). Manag Health. 13, 18-21 (2009)

13. Griffiths, R.I., Gleeson, M.L., Mikhael, J., Dreyling, M.H., Danese, M.D.: Comparative effectiveness and cost of adding rituximab to first-line chemotherapy for elderly patients diagnosed with diffuse large B-cell lymphoma. Cancer 118, 6079-6088 (2012)

14. Best, J.H., Hornberger, J., Proctor, S.J., Omnes, L.F., Jost, F.: Cost-effectiveness analysis of rituximab combined with chop for treatment of diffuse large B-cell lymphoma. Value Health. 8, 462-470 (2005)

15. Groot, M.T., Lugtenburg, P.J., Hornberger, J., Huijgens, P.C., Uyl-de Groot, C.A.: Cost-effectiveness of rituximab (MabThera) in diffuse large B-cell lymphoma in the Netherlands. Eur. J. Haematol. 74, 194-202 (2005)

16. Hornberger, J.C., Best, J.H.: Cost utility in the United States of rituximab plus cyclophosphamide, doxorubicin, vincristine, and prednisone for the treatment of elderly patients with diffuse large B-cell lymphoma. Cancer 103, 1644-1651 (2005)

17. Lee, R.C., Zou, D., Demetrick, D.J., Difrancesco, L.M., Fassbender, K., Stewart, D.: Costs associated with diffuse large B-cell lymphoma patient treatment in a Canadian integrated cancer care center. Value Health. 11, 221-230 (2008)

18. Wang, H.-I., Aas, E., Howell, D., Roman, E., Patmore, R., Jack, A., Smith, A.: Long-term medical costs and life expectancy of acute myeloid leukemia: a probabilistic decision model. Value Health. 17, 205-214 (2014)

19. Payment by results in the NHS: tariff for 2013-2014. https:// www.gov.uk/government/publications/payment-by-results-pbroperational-guidance-and-tariffs (2013)

20. NHS reference costs 2012-2013. https://www.gov.uk/govern ment/publications/nhs-reference-costs-2012-to-2013 (2013) 
21. United Kingdom, National Life Tables, 1980-82 to 2011-13. http://www.ons.gov.uk/ons/publications/re-reference-tables.html? edition=tcm\%3A77-365199

22. Guide to the methods of technology appraisal 2008. http://www. nice.org.uk/media/B52/A7/TAMethodsGuideUpdatedJune2008. pdf (2012)

23. Briggs, A., Sculpher, M., Claxton, K.: Decision modelling for health economic evaluation. Oxford University Press (2006)

24. Eddy, D.M., Hollingworth, W., Caro, J.J., Tsevat, J., McDonald, K.M., Wong, J.B.: Model transparency and validation: a report of the ISPOR-SMDM modeling good research practices task force7. Value. Health. 15, 843-850 (2012)

25. Smith, A., Crouch, S., Howell, D., Burton, C., Patmore, R., Roman, E.: Impact of age and socioeconomic status on treatment and survival from aggressive lymphoma: a UK population-based study of diffuse large B-cell lymphoma. Cancer Epidemiol. (2015)

26. 2012-13 Programme budgeting data. http://www.england.nhs.uk/ resources/resources-for-ccgs/prog-budgeting/ (2013)

27. Camicia, R., Winkler, H.C., Hassa, P.O.: Novel drug targets for personalized precision medicine in relapsed/refractory diffuse large B-cell lymphoma: a comprehensive review. Mol. Cancer. 14, 207 (2015)

28. Roschewski, M., Staudt, L.M., Wilson, W.H.: Diffuse large B-cell lymphoma-treatment approaches in the molecular era. Nat Rev Clin Oncol. 11, 12-23 (2014)

29. UK Clinical Research Network: REMoDLB. UK Clinical Research Network 